T. Kubota

Nagoya Math. J.

Vol. 127 (1992), 61-82

\title{
AUTOMORPHIC FORMS AND INFINITE MATRICES
}

\author{
TOMIO KUBOTA
}

In the present paper, we show that an infinite dimensional vector whose components are Fourier coefficients of an automorphic form is characterized as an infinite dimensional vector which is annihilated by an infinite matrix constructed by the values of a Bessel function. Results and methods are all simple and concrete.

Although the idea in the present paper is applicable to more general cases, our investigation will be restricted to the case of automorphic forms of weight 0 , i.e., automorphic functions, with respect to $\operatorname{SL}(2, \mathbf{Z})$ on the upper half plane, in order to explain the main idea distinctly.

The main theorem is stated in connection with cusp forms in Section 3. In Section 4 , we note first that the main theorem is a characterization of eigenvalues of the Laplacian, and show that the eigenvalues of the Laplacian and the zeros of the Riemann zeta function are characterized simultaneously by extending the range of functions slightly out of cusp forms. This fact can be regarded as an example of direct and naive contacts between infinite matrices and zeros of zeta functions.

The most essential part of the present paper consists of approximation formulas proved in Section 2. Because of such formulas, the condition for the components of an infinite vector to be Fourier coefficients of an automorphic form is, as seen for instance in the main theorem, reduced to the sole assertion that the vector is annihilated by an infinite matrix.

\section{§1. Preliminaries}

In this section, we recall some basic notions, prepare symbols, and prove Theorem 1.1. The main theorem of the present paper (Theorem 3.1) is the converse of Theorem 1.1 .

In the sequel, an automorphic form means a function on the upper half plane $S=\{z \in \mathbf{C} ; \operatorname{Im} z>0\}$ which is invariant under the linear transformation Received January 22. 1992. 
$z \rightarrow \sigma z=\frac{a z+b}{c z+d}$ determined by an element $\sigma=\left(\begin{array}{ll}a & b \\ c & d\end{array}\right)$ of SL(2, Z), is an eigenfunction of the Laplacian

$$
D=y^{2}\left(\frac{\partial^{2}}{\partial x^{2}}+\frac{\partial^{2}}{\partial y^{2}}\right), \quad(z=x+i y),
$$

of $S$, and is slowly increasing as $y \rightarrow \infty$. The eigenvalue will be expressed by an $s$ $\in \mathbf{C}$ with

$$
D f=s(s-1) f .
$$

This says that $f$ belongs to the eigenvalue $s(s-1)$, but, for the sake of simplicity, we say in this case that $f$ belongs to $s$. Besides, we put

$$
s=\sigma+i t, \quad(\sigma, t \in \mathbf{R}) .
$$

Since an automorphic form $f$ has the period 1 with respect to $x$, it is expanded into a Fourier series in $e(m x),(m \in \mathbf{Z})$, with

$$
e(x)=\exp (2 \pi i x) .
$$

In general, the Fourier series takes the form

$$
f(z)=c_{0}^{\prime} y^{s}+c_{0} y^{1-s}+\sum_{m \neq 0} c_{m} y^{\frac{1}{2}} K_{s-\frac{1}{2}}(2 \pi|m| y) e(m x),
$$

where $K$ is a modified Bessel function, and any function with this Fourier expansion is $O\left(y^{\max (\sigma, 1-\sigma)}\right)$ so that it is slowly increasing as $y \rightarrow \infty$.

An automorphic form $f(z)$ is called a cusp form, if $c_{0}^{\prime}=c_{0}=0$. If $f(z)$ is an even function with respect to $x$, i.e., if $f(z)=f(-\bar{z})$, then $f$ is called even. The Fourier expansion of an even cusp form $f$ is of the form

$$
f(z)=\sum_{m=1}^{\infty} c_{m} y^{\frac{1}{2}} K_{s-\frac{1}{2}}(2 \pi m y) \cos 2 \pi m x .
$$

For later use, we pick up here some asymptotic formulas concerning the Bessel function $K$.

$$
\begin{gathered}
K_{s-\frac{1}{2}}(2 \pi y)=\frac{1}{2} y^{-\frac{1}{2}} \exp (-2 \pi y)\left(1+O\left(y^{-1}\right)\right), \quad(y \rightarrow \infty), \\
K_{s-\frac{1}{2}}(2 \pi y)=\alpha\left(s-\frac{1}{2}\right) y^{s-\frac{1}{2}}+\alpha\left(-s+\frac{1}{2}\right) y^{-s+\frac{1}{2}} \\
+O\left(y^{-\sigma+\frac{5}{2}}\right), \quad\left(\sigma \geqq \frac{1}{2}, s \neq \frac{1}{2}, y \rightarrow 0\right), \\
K_{0}(2 \pi y)=-\log y+O(1),(y \rightarrow 0)
\end{gathered}
$$

where the implied constants in (4) and (5) depend on $s$, and 


$$
\alpha(s)= \begin{cases}0, & \text { if } s \in \mathbf{Z} \text { and } s>0, \\ \frac{1}{2} \pi^{s} \Gamma(-s), & \text { otherwise. }\end{cases}
$$

We also refer to a well-known proposition.

PROPOSITION 1.1. Let

$$
f(z)=\sum_{m \neq 0} c_{m} y^{\frac{1}{2}} K_{s-\frac{1}{2}}(2 \pi|m| y) e(m x)
$$

be a cusp form. Then, $c_{m}=O\left(|m|^{\frac{1}{2}}\right)$.

Proof. Since $f(z)$ is bounded on the whole upper half plane $S$, there exists a constant $M$ such that $|f(z)|<M$. Put now $y=\alpha_{0}|m|^{-1},\left(K_{s-\frac{1}{2}}\left(2 \pi \alpha_{0}\right) \neq 0\right)$. Then,

$$
\int_{0}^{1} f\left(x+\frac{i \alpha_{0}}{|m|}\right) e(-m x) d x=\alpha_{0}^{\frac{1}{2}} c_{m}|m|^{-\frac{1}{2}} K_{s-\frac{1}{2}}\left(2 \pi \alpha_{0}\right)
$$

implies

$$
\left|c_{m}\right|<\alpha_{0}^{-\frac{1}{2}} M\left|K_{s-\frac{1}{2}}\left(2 \pi \alpha_{0}\right) \| m\right|^{\frac{1}{2}}
$$

Remark 1.1. If $f$ is a general automorphic form as in (2), and if $\sigma=\operatorname{Re} s$ $\geqq \frac{1}{2}$, then $|f(z)|<M y^{-\sigma}$, and so

$$
\left|\int_{0}^{1} f\left(x+\frac{i \alpha_{0}}{|m|}\right) e(-m x) d x\right|<\alpha_{0}^{-\sigma} M|m|^{\sigma} .
$$

This implies $c_{m}=O\left(|m|^{\frac{1}{2}+\sigma}\right)$ for $\sigma \geqq \frac{1}{2}$. More generally, we have

$$
c_{m}=O\left(|m|^{\frac{1}{2}+\max (\sigma, 1-\sigma)}\right)
$$

for an arbitrary $\sigma$.

For two complex valued functions $f_{1}$ and $f_{2}$ of a positive, real variable $y$, an inner product $\left\langle f_{1}, f_{2}\right\rangle$ is defined by

$$
\left\langle f_{1}, f_{2}\right\rangle=\int_{0}^{\infty} \overline{f_{1}(y)} f_{2}(y) \omega(y) \frac{d y}{y}
$$

with 


$$
\omega(y)=\exp \left(-2 \pi\left(y+y^{-1}\right)\right) .
$$

This inner product defines a Hilbert space which will be denoted by $\Omega$. Furthermore, we put

$$
g_{m}(y)=y^{\frac{1}{2}} K_{s-\frac{1}{2}}(2 \pi m y)-y^{-\frac{1}{2}} K_{s-\frac{1}{2}}\left(2 \pi m y^{-1}\right),
$$

$$
h(l, m)=\left\langle g_{l}, g_{m}\right\rangle,
$$

$(l, m=1,2,3, \ldots)$.

PROPOSITION 1.2. For any $\gamma$ with $0<\gamma<1$, there exists a constant $C>0$, depending on $r$ and $s$, such that

$$
|h(l, m)| \leqq\left\langle\left|g_{l}\right|,\left|g_{m}\right|\right\rangle\left\langle C \exp \left(-4 \pi \gamma(l+m)^{\frac{1}{2}}\right)\right.
$$

holds for all $l, m=1,2, \ldots$.

Proof. We may assume $\sigma \geqq \frac{1}{2}$ since $K_{\nu}=K_{-\nu}$. It follows from (4), (5), and (6) that there exists a constant $C_{1}$, depending on $\gamma$ and $s$, such that

$$
\left|y^{\frac{1}{2}} K_{s-\frac{1}{2}}(2 \pi y)\right|<C_{1} y^{\frac{1}{2}-\sigma} \exp (-2 \pi r y)
$$

holds for all $y$. Therefore we have

$$
\begin{gathered}
\left|y^{\frac{1}{2}} K_{s-\frac{1}{2}}(2 \pi m y)\right|<C_{1} m^{-\frac{1}{2}}(m y)^{\frac{1}{2}-\sigma} \exp (-2 \pi r m y), \\
\left|y^{-\frac{1}{2}} K_{s-\frac{1}{2}}\left(2 \pi m y^{-1}\right)\right|<C_{1} m^{-\frac{1}{2}}\left(m y^{-1}\right)^{\frac{1}{2}-\sigma} \exp \left(-2 \pi r m y^{-1}\right) .
\end{gathered}
$$

Hence

$$
\left|g_{m}(y)\right|<C_{1} m^{-\sigma}\left(y^{\frac{1}{2}-\sigma} \exp (-2 \pi \gamma m y)+y^{\frac{1}{2}-\sigma} \exp \left(-2 \pi \gamma m y^{-1}\right)\right),
$$

This implies

$$
\begin{aligned}
& \left|g_{l}(y) g_{m}(y) \omega(y)\right|<C_{1}^{2}(l m)^{-\sigma}\left[y^{1-2 \sigma} \exp (-2 \pi \gamma(l+m) y)\right. \\
& +\exp \left(-2 \pi \gamma\left(l y+m y^{-1}\right)\right)+\exp \left(-2 \pi \gamma\left(l y^{-1}+m y\right)\right) \\
& \left.+y^{-1+2 \sigma} \exp \left(-2 \pi \gamma(l+m) y^{-1}\right)\right] \omega(y) \\
& =C_{1}^{2}(l m)^{-\sigma}\left[y^{1-2 \sigma} \exp \left(-2 \pi\left((\gamma l+\gamma m+1) y+y^{-1}\right)\right)\right. \\
& \quad+\exp \left(-2 \pi\left((\gamma l+1) y+(\gamma m+1) y^{-1}\right)\right) \\
& +\exp \left(-2 \pi\left((\gamma m+1) y+(\gamma l+1) y^{-1}\right)\right) \\
& \left.+y^{-1+2 \sigma} \exp \left(-2 \pi\left(y+(\gamma l+\gamma m+1) y^{-1}\right)\right)\right] .
\end{aligned}
$$


Let us recall here the integral formula

$$
\int_{0}^{\infty} \exp \left(-2 \pi\left(l^{\prime} y+m^{\prime} y^{-1}\right)\right) y^{\nu} \frac{d y}{y}=2\left(\frac{m^{\prime}}{l^{\prime}}\right)^{\frac{\nu}{2}} K_{\nu}\left(4 \pi\left(l^{\prime} m^{\prime}\right)^{\frac{1}{2}}\right)
$$

$\left(l^{\prime}, m^{\prime}>0\right)$, to obtain

$$
\begin{gathered}
\left\langle\left|g_{l}\right|,\left|g_{m}\right|\right\rangle \leqq \int_{0}^{\infty}\left|g_{l}(y) g_{m}(y)\right| \omega(y) \frac{d y}{y} \\
<4 C_{1}^{2}(l m)^{-\sigma}\left[(\gamma l+\gamma m+1)^{-\frac{1}{2}+\sigma} K_{2 \sigma-1}\left(4 \pi(\gamma l+\gamma m+1)^{\frac{1}{2}}\right)\right. \\
+K_{0}\left(4 \pi(\gamma l+1)^{\left.\left.\frac{1}{2}(\gamma m+1)^{\frac{1}{2}}\right)\right] .}\right.
\end{gathered}
$$

Then, applying again the asymptotic formula (4) to the Bessel functions in the above formula, we have the evaluation

$$
\begin{gathered}
\left\langle\left|g_{l}\right|,\left|g_{m}\right|\right\rangle \\
\left\langle C_{2}\left[\exp \left(-4 \pi(\gamma l+\gamma m+1)^{\frac{1}{2}}\right)+\exp \left(-4 \pi(\gamma l+1)^{\frac{1}{2}}(\gamma m+1)^{\frac{1}{2}}\right)\right]\right.
\end{gathered}
$$

with a constant $C_{2}$. Now,

$$
(\gamma l+1)(\gamma m+1)>\gamma(l+m)>\gamma^{2}(l+m)
$$

yields

$$
\left\langle\left|g_{l}\right|,\left|g_{m}\right|\right\rangle\left\langle C \exp \left(-4 \pi r(l+m)^{\frac{1}{2}}\right) .\right.
$$

If $l$ and $m$ are regarded as indices of rows and columns, respectively, then $\left\langle g_{l}, g_{m}\right\rangle$ forms an infinite matrix $H=H(s)$, i.e.,

$$
H=H(s)=\left(\left\langle g_{l}, g_{m}\right\rangle\right)=(h(l, m))_{l, m=1}^{\infty} .
$$

This matrix $H$ is hermitian.

THEOREM 1.1. Let

$$
f(z)=\sum_{m=1}^{\infty} c_{m} y^{\frac{1}{2}} K_{s-\frac{1}{2}}(2 \pi m y) \cos 2 \pi m x
$$

be an even cusp form, and let $c_{*}$ be an infinite column vector whose $m$-th component is $c_{m}$. Then,

$$
H c_{*}=0
$$

holds in the sense that $\sum_{m=1}^{\infty} h(l, m) c_{m}$ converges to 0 for every $l$. 
Proof. Fix $l$ arbitrarily. Then, because of Propositions 1.1 and 1.2, $\sum_{m=1}^{\infty}\left\langle\left|g_{l}\right|,\left|g_{m}\right|\right\rangle\left|c_{m}\right|$ converges. This means the convergence of

$$
\sum_{m=1}^{\infty} \int_{0}^{\infty}\left|g_{l}(y) \cdot c_{m} g_{m}(y)\right| \omega(y) \frac{d y}{y}
$$

and therefore

$$
\sum_{m=1}^{\infty} h(l, m) c_{m}=\int_{0}^{\infty} \overline{g_{l}(y)}\left(\sum_{m=1}^{\infty} c_{m} g_{m}(y)\right) \omega(y) \frac{d y}{y} .
$$

Since $\sum_{m=1}^{\infty} c_{m} g_{m}(y)=f(i y)-f\left(i y^{-1}\right)=0$, we have

$$
\sum_{m=1}^{\infty} h(l, m) c_{m}=0
$$

\section{§ 2. Determination of the vanishing orders of the entries of $H$}

To apply to the next section, we now propose to determine the vanishing order of elements $h(l, m)$ of $H$ correctly for a fixed $l$ as $m \rightarrow \infty$, not as in Proposition 1.2, where they were evaluated from above. The implied constants in $O$-symbols in the statements of propositions in the sequel depend on $s$ and on an additional parameter $\beta$.

For $l, m>0$ and $s \in \mathbf{C}$, put

$$
\begin{aligned}
& h_{1}(l, m)=\int_{0}^{\infty} K_{s-\frac{1}{2}}(2 \pi l y) K_{s-\frac{1}{2}}(2 \pi m y) \omega(y) y \frac{d y}{y}, \\
& h_{2}(l, m)=\int_{0}^{\infty} K_{\bar{s}-\frac{1}{2}}\left(2 \pi l y^{-1}\right) K_{s-\frac{1}{2}}(2 \pi m y) \omega(y) \frac{d y}{y},
\end{aligned}
$$

where $\omega$ is as in (8). Then,

$$
h(l, m)=2 h_{1}(l, m)-2 h_{2}(l, m)
$$

follows from the definition $(10)$ of $h(l, m)$.

Proposition 2.1. Let $\alpha(s)$ be as in (7), and assume $\beta \in \mathbf{C}, s, s^{\prime} \in \mathbf{C}$, and $s \neq \frac{1}{2}$. Then,

$$
\begin{gathered}
\int_{0}^{\infty} K_{s-\frac{1}{2}}(2 \pi l y) K_{s^{\prime}-\frac{1}{2}}(2 \pi m y) \omega(y) y^{\beta} d y \\
=2^{-\frac{3}{2}}\left(\alpha\left(s-\frac{1}{2}\right) l^{s-\frac{1}{2}} m^{\frac{1}{2}\left(-s+\frac{1}{2}\right)-\frac{\beta+2}{2}}\right.
\end{gathered}
$$




$$
\left.+\alpha\left(-s+\frac{1}{2}\right) l^{-s+\frac{1}{2}} m^{\frac{1}{2}\left(s-\frac{1}{2}\right)-\frac{\beta+2}{2}}\right) \exp \left(-4 \pi m^{\frac{1}{2}}\right)\left(1+O\left(m^{-\frac{1}{2}}\right)\right)
$$

holds for a fixed $l$ as $m \rightarrow \infty$.

Proof. Throughout the proof, we assume $l \leqq m$.

Put first

$$
A=\int_{0}^{\infty} K_{s-\frac{1}{2}}(2 \pi l y) K_{s^{\prime}-\frac{1}{2}}(2 \pi m y) \omega(y) y^{\beta} d y
$$

and apply (4), (5) and (6) to

$$
A=\int_{0}^{\frac{1}{m}}+\int_{\frac{1}{m}}^{\frac{1}{l}}+\int_{\frac{1}{l}}^{\infty}
$$

Then, in case $s^{\prime} \neq \frac{1}{2}$, we have

$$
\begin{gathered}
A=\int_{0}^{\frac{1}{m}}\left(\alpha\left(s-\frac{1}{2}\right)(l y)^{s-\frac{1}{2}}+\alpha\left(-s+\frac{1}{2}\right)(l y)^{-s+\frac{1}{2}}\right)\left(1+O\left((l y)^{2}\right)\right) . \\
\left.\cdot \alpha\left(s^{\prime}-\frac{1}{2}\right)(m y)^{s^{\prime}-\frac{1}{2}}+\alpha\left(-s^{\prime}+\frac{1}{2}\right)(m y)^{-s^{\prime}+\frac{1}{2}}\right)\left(1+O\left((m y)^{2}\right)\right) \omega(y) y^{\beta} d y \\
\left.+\int_{\frac{1}{m}}^{\frac{1}{l}} \alpha\left(s-\frac{1}{2}\right)(l y)^{s-\frac{1}{2}}+\alpha\left(-s+\frac{1}{2}\right)(l y)^{-s+\frac{1}{2}}\right)\left(1+O\left((l y)^{2}\right)\right) . \\
\cdot \frac{1}{2}(m y)^{-\frac{1}{2}} \exp (-2 \pi m y)\left(1+O\left((m y)^{-1}\right)\right) \omega(y) y^{\beta} d y \\
+\int_{\frac{1}{l}}^{\infty} \frac{1}{2}(l y)^{-\frac{1}{2}} \exp (-2 \pi l y)\left(1+O\left((l y)^{-1}\right)\right) . \\
\cdot \frac{1}{2}(m y)^{-\frac{1}{2}} \exp (-2 \pi m y)\left(1+O\left((m y)^{-1}\right)\right) \omega(y) y^{\beta} d y .
\end{gathered}
$$

In case $s^{\prime}=\frac{1}{2},(6)$ is used to deform the first integral so that the result is somewhat different. But, in either case,

$$
\omega(y)<\exp \left(-2 \pi y^{-1}\right)
$$

yields the fact that the first integral is $O\left(l^{\left|\sigma-\frac{1}{2}\right|} \exp (-2 \pi \gamma m)\right),(\sigma=\operatorname{Re} s)$, where $\gamma$ is an arbitrary real number with $0<\gamma<1$. On the other hand, the third integral is $O\left(\exp \left(-2 \pi r \frac{m}{l}\right)\right)$, since

$$
\int_{\frac{1}{l}}^{\infty} \exp (-2 \pi m y) d y=\frac{1}{2 \pi m} \exp \left(-2 \pi \frac{m}{l}\right) .
$$

Thus, $A$ is expressed in the form 


$$
\begin{aligned}
& \left.A=\int_{\frac{1}{m}}^{\frac{1}{l}} \alpha\left(s-\frac{1}{2}\right)(l y)^{s-\frac{1}{2}}+\alpha\left(-s+\frac{1}{2}\right)(l y)^{-s+\frac{1}{2}}\right)\left(1+P\left((l y)^{2}\right)\right) \cdot \\
& \quad \cdot \frac{1}{2}(m y)^{-\frac{1}{2}} \exp (-2 \pi m y)\left(1+Q\left((m y)^{-1}\right)\right) \omega(y) y^{\beta} d y \\
& +O\left(\exp \left(-2 \pi r \frac{m}{l}\right)\right),
\end{aligned}
$$

leaving solely the second integral. Here, $P$ and $Q$ are functions expressing two parts which formerly written as $O\left((l y)^{2}\right)$ and $O\left((m y)^{-1}\right)$, respectively. Since these functions $P$ and $Q$ are hitherto defined and used only on the closed intervals $\left[\frac{l^{2}}{m^{2}}, 1\right]$ and $\left[\frac{l}{m}, 1\right]$, respectively, it has nothing to do with the above expression of $A$ to extend $P$ and $Q$ beyond the intervals. Therefore, we assume now that $P$ and $Q$ are defined for all $y>0$ and satisfy

$$
|P(y)|<C_{3} y, \quad|Q(y)|<C_{4} y,
$$

where $C_{3}$ and $C_{4}$ are constants depending on $s$ and $s^{\prime}$, respectively. In the same way as the estimation of the error term in the above expression of $A$, we see that the change of the value is only $O\left(\exp \left(-2 \pi r \frac{m}{l}\right)\right)$ if $\int_{\frac{1}{m}}^{\frac{1}{l}}$ in the expression is replaced by $\int_{0}^{\infty}$. Hence.

$$
\begin{aligned}
& A=\int_{0}^{\infty}\left(\alpha\left(s-\frac{1}{2}\right)(l y)^{s-\frac{1}{2}}+\alpha\left(-s+\frac{1}{2}\right)(l y)^{-s+\frac{1}{2}}\right)\left(1+P\left((l y)^{2}\right)\right) . \\
& \quad \cdot \frac{1}{2}(m y)^{-\frac{1}{2}} \exp (-2 \pi m y)\left(1+Q\left((m y)^{-1}\right)\right) \omega(y) y^{\beta} d y \\
& +O\left(\exp \left(-2 \pi r \frac{m}{l}\right)\right) .
\end{aligned}
$$

Divide the integral in this formula into four parts which are contributions of terms in the expansion

$$
(1+P)(1+Q)=1+P+Q+P Q,
$$

and denote them $A_{1}, A_{P}, A_{Q}$, and $A_{P Q}$, respectively. Then, by putting $\beta^{\prime}=$ $\pm\left(s+\frac{1}{2}\right)-\frac{1}{2}+\beta$ in the formula

$$
\begin{gathered}
\int_{0}^{\infty} \exp (-2 \pi m y) \omega(y) y^{\beta^{\prime}} d y=\int_{0}^{\infty} \exp \left(-2 \pi\left((m+1) y+y^{-1}\right)\right) y^{\beta^{\prime}} d y \\
=2(m+1)^{-\frac{1}{2}\left(\beta^{\prime}+1\right)} K_{\beta^{\prime}+1}\left(4 \pi(m+1)^{\frac{1}{2}}\right)
\end{gathered}
$$

derived from the integral formula (11), $A_{1}$ takes the expression 


$$
\begin{aligned}
& A_{1}=m^{-\frac{1}{2}}\left(\alpha\left(s-\frac{1}{2}\right) l^{s-\frac{1}{2}}(m+1)^{-\frac{1}{2}(s+\beta)} K_{s+\beta}\left(4 \pi(m+1)^{\frac{1}{2}}\right)\right. \\
& \left.+\alpha\left(-s+\frac{1}{2}\right) l^{-s+\frac{1}{2}}(m+1)^{-\frac{1}{2}(-s+\beta+1)} K_{-s+\beta+1}\left(4 \pi(m+1)^{\frac{1}{2}}\right)\right) .
\end{aligned}
$$

As for $A_{P}, A_{Q}$, and $A_{P Q}$, computations of the same kind using (16) lead to the evaluations

$$
\begin{gathered}
\left|A_{P}\right|<C_{3} l^{2} A_{1}^{*}(\beta+2), \\
\left|A_{Q}\right|<C_{4} m^{-1} A_{1}^{*}(\beta-1), \\
\left|A_{P Q}\right|<C_{3} C_{4} l^{2} m^{-1} A_{1}^{*}(\beta+1)
\end{gathered}
$$

with

$$
\begin{aligned}
& A_{1}^{*}(\beta)=m^{-\frac{1}{2}}\left(\left|\alpha\left(s-\frac{1}{2}\right)\right| l^{\sigma-\frac{1}{2}}(m+1)^{-\frac{1}{2}(\sigma+\beta)} K_{\sigma+\beta}\left(4 \pi(m+1)^{\frac{1}{2}}\right)\right. \\
& \left.+\left|\alpha\left(-s+\frac{1}{2}\right)\right| l^{-\sigma+\frac{1}{2}}(m+1)^{-\frac{1}{2}(-\sigma+\beta+1)} K_{-\sigma+\beta+1}\left(4 \pi(m+1)^{\frac{1}{2}}\right)\right) .
\end{aligned}
$$

If (4) is applied to (19), then there appears the formula in the proposition, and the order of $m$ in the formula is higher than the order of $m$ in the error term of (17). Furthermore, (20) shows that the orders of $m$ in $A_{P}, A_{Q}$, and in $A_{P Q}$ are lower than the order of $m$ in $A_{1}$ at least by $m^{\frac{1}{2}}$. This completes the proof.

If $s=\frac{1}{2}$, then (6) must be used instead of (5). Accordingly it turns out to be difficult to obtain a result corresponding to this proposition. For our purpose, however, the following asymptotic inequality is enough:

Proposition 2.2. Let $\beta \in \mathbf{R}$ and $l>0$. Then, the inequality

$$
\int_{0}^{\infty} K_{0}(2 \pi l y) K_{0}(2 \pi m y) \omega(y) y^{\beta} d y>C_{0} m^{-\frac{\beta+2}{2}} \exp \left(-4 \pi m^{\frac{1}{2}}\right)
$$

holds for all sufficiently large $m>0$, where $C_{0}$ is an absolute constant.

Proof. Denote by $A_{0}$ the integral in question, assume $m \geqq l$ and divide $A_{0}$ as

$$
A_{0}=\int_{0}^{\frac{1}{m}}+\int_{\frac{1}{m}}^{\frac{1}{l}}+\int_{\frac{1}{l}}^{\infty} .
$$

Then, since the integrals are positive, we have $A_{0}>\int_{\frac{1}{m}}^{\frac{1}{l}}$. Next, the monotonous decreasingness of $K_{0}$ entails

$$
\int_{\frac{1}{m}}^{\frac{1}{l}}>\int_{\frac{1}{m}}^{\frac{1}{l}} K_{0}(2 \pi m y) \omega(y) y^{\beta} d y
$$


and, by means of (4), the last integral is expressed as

$$
K_{0}(2 \pi) \int_{\frac{1}{m}}^{\frac{1}{l}} \frac{1}{2}(m y)^{-\frac{1}{2}} \exp (-2 \pi m y)\left(1+Q\left((m y)^{-1}\right)\right) \omega(y) y^{\beta} d y,
$$

where $Q$ is as in (16) with $s^{\prime}=\frac{1}{2}$. The change of the value is $O\left(\exp \left(-2 \pi r \frac{m}{l}\right)\right)$ in this case, when $\int_{\frac{1}{m}}^{\frac{1}{l}}$ is replaced by $\int_{0}^{\infty}$. Therefore we have

$$
\begin{aligned}
A_{0}>K_{0}(2 \pi) \int_{0}^{\infty} \frac{1}{2}(m y)^{-\frac{1}{2}} & \exp (-2 \pi m y)\left(1+Q\left((m y)^{-1}\right)\right) \omega(y) y^{\beta} d y \\
+ & O\left(\exp \left(-2 \pi r \frac{m}{l}\right)\right) .
\end{aligned}
$$

Divide the above integral into two parts which are contributions of terms in the sum $1+Q$, and denote them by $A_{0,1}$ and $A_{0,2}$, respectively. Then, (18) with $\beta^{\prime}=$ $\beta-\frac{1}{2}$ yields

$$
A_{0,1}=m^{-\frac{1}{2}}(m+1)^{-\frac{1}{2}\left(\beta+\frac{1}{2}\right)} K_{\beta+\frac{1}{2}}\left(4 \pi(m+1)^{\frac{1}{2}}\right)
$$

and this is $2^{-\frac{3}{2}} m^{-\frac{\beta+2}{2}} \exp \left(-4 \pi m^{\frac{1}{2}}\right)\left(1+O\left(m^{-\frac{1}{2}}\right)\right)$. On the other hand, a computa tion with $\beta^{\prime}=\beta-\frac{3}{2}$ shows that the order of $m$ in $A_{0, Q}$ is lower than the order of $m$ in $A_{0,1}$ by $\frac{1}{2}$. Thus, the proposition is valid for any $C_{0}$ with $0<C_{0}<$ $2^{-\frac{3}{2}} K_{0}(2 \pi)$.

Proposition 2.3. Let $\beta \in \mathbf{C}$ and $s, s^{\prime} \in \mathbf{C}$. Then,

$$
\begin{aligned}
& \int_{0}^{\infty} K_{s-\frac{1}{2}}\left(2 \pi l y^{-1}\right) K_{s^{\prime}-\frac{1}{2}}(2 \pi m y) \omega(y) y^{\beta} d y \\
& =2^{-\frac{5}{2}} l^{-\frac{1}{2}}(l+1)^{\frac{1}{2} \beta+\frac{1}{4}} m^{-\frac{1}{2} \beta-\frac{5}{4}} \exp \left(-4 \pi(l+1)^{\frac{1}{2}} m^{\frac{1}{2}}\right)\left(1+O\left(m^{-\frac{1}{2}}\right)\right)
\end{aligned}
$$

holds for a fixed $l>0$ as $m \rightarrow \infty$.

Proof. We always assume $m \geqq l$.

Denote by $A^{\prime}$ the integral in the proposition, i.e.,

$$
A^{\prime}=\int_{0}^{\infty} K_{s-\frac{1}{2}}\left(2 \pi l y^{-1}\right) K_{s^{\prime}-\frac{1}{2}}(2 \pi m y) \omega(y) y^{\beta} d y .
$$

Divide $A^{\prime}$ as 


$$
A^{\prime}=\int_{0}^{\frac{1}{m}}+\int_{\frac{1}{m}}^{l}+\int_{l}^{m}
$$

and assume first $s^{\prime} \neq \frac{1}{2}, s \neq \frac{1}{2}$. Then, it follows from (4), (5), and (6) that

$$
\begin{gathered}
A^{\prime}=\int_{0}^{\frac{1}{m}} \frac{1}{2}\left(l y^{-1}\right)^{-\frac{1}{2}} \exp \left(-2 \pi l y^{-1}\right)\left(1+O\left(\left(l y^{-1}\right)^{-1}\right)\right) \cdot \\
\cdot\left(\alpha\left(s^{\prime}-\frac{1}{2}\right)(m y)^{s^{\prime}-\frac{1}{2}}+\alpha\left(-s^{\prime}+\frac{1}{2}\right)(m y)^{-s^{\prime}+\frac{1}{2}}\right)\left(1+O\left((m y)^{2}\right)\right) \omega(y) y^{\beta} d y \\
+\int_{\frac{1}{m}}^{l} \frac{1}{2}\left(l y^{-1}\right)^{-\frac{1}{2}} \exp \left(-2 \pi l y^{-1}\right)\left(1+O\left(\left(l y^{-1}\right)^{-1}\right)\right) \cdot \\
\cdot \frac{1}{2}(m y)^{-\frac{1}{2}} \exp (-2 \pi m y)\left(1+O\left((m y)^{-1}\right)\right) \omega(y) y^{\beta} d y \\
+\int_{l}^{\infty}\left(\alpha\left(s-\frac{1}{2}\right)\left(l y^{-1}\right)^{s-\frac{1}{2}}+a\left(-s+\frac{1}{2}\right)\left(l y^{-1}\right)^{-s+\frac{1}{2}}\right)\left(1+O\left(\left(l y^{-1}\right)^{2}\right)\right) . \\
\cdot \frac{1}{2}(m y)^{-\frac{1}{2}}+\exp (-2 \pi m y)\left(1+O\left((m y)^{-1}\right)\right) \omega(y) y^{\beta} d y .
\end{gathered}
$$

If either $s$ or $s^{\prime}$ is equal to $\frac{1}{2}$, then (6) is used to deform the first and the third integrals so that the results are somewhat different. But, in either case, the first integral is $O(\exp (-2 \pi r m))$ because of $\omega(y)<\exp \left(-2 \pi y^{-1}\right)$, where $\gamma$ is an arbitrary real number with $0<r<1$. On the other hand, the third integral is $O(\exp (-2 \pi r m))$ because of

$$
\int_{l}^{\infty} \exp (-2 \pi m y) d y=\frac{1}{2 \pi m} \exp (-2 \pi l m) .
$$

Therefore, leaving solely the second integral, $A^{\prime}$ is expressed as

$$
\begin{gathered}
A^{\prime}=\int_{\frac{1}{m}}^{l} \frac{1}{2}\left(l y^{-1}\right)^{-\frac{1}{2}} \exp \left(-2 \pi l y^{-1}\right)\left(1+\widetilde{Q}\left(\left(l y^{-1}\right)^{-1}\right)\right) \cdot \\
\cdot \frac{1}{2}(m y)^{-\frac{1}{2}} \exp (-2 \pi m y)\left(1+Q\left((m y)^{-1}\right)\right) \omega(y) y^{\beta} d y+O(\exp (-2 \pi r m)) .
\end{gathered}
$$

Here, $Q$ is as in (16), and $\widetilde{Q}$ is obtained from $Q$ by replacing the variable $s^{\prime}$ with $s$, i.e.,

$$
\widetilde{Q}=\left.Q\right|_{s^{\prime} \rightarrow s} .
$$

In the same way as the estimation of the error term in the above fromula, it is shown that the change of value is also $O(\exp (-2 \pi r m))$ if the integral $\int_{\frac{1}{m}}^{l}$ in the above formula is replaced by $\int_{0}^{\infty}$ 
Hence,

$$
\begin{gathered}
A^{\prime}=\int_{0}^{\infty} \frac{1}{2}\left(l y^{-1}\right)^{-\frac{1}{2}} \exp \left(-2 \pi l y^{-1}\right)\left(1+\widetilde{Q}\left(\left(l y^{-1}\right)^{-1}\right)\right) \cdot \\
\cdot \frac{1}{2}(m y)^{-\frac{1}{2}} \exp (-2 \pi m y)\left(1+Q\left((m y)^{-1}\right)\right) \omega(y) y^{\beta} d y+O(\exp (-2 \pi r m)) .
\end{gathered}
$$

Divide this integral into four parts which are contributions of terms in the expansion

$$
(1+\widetilde{Q})(1+Q)=1+\widetilde{Q}+Q+\widetilde{Q} Q,
$$

and denote them by $A_{1}^{\prime}, A_{\tilde{Q}}^{\prime}, A_{Q}^{\prime}$, and $A_{\tilde{Q} Q}^{\prime}$, respectively. Then, (11) implies in general

$$
\begin{aligned}
& \int_{0}^{\infty} \exp \left(-2 \pi\left(m y+l y^{-1}\right)\right) \omega(y) y^{\beta^{\prime}} d y \\
= & \int_{0}^{\infty} \exp \left(-2 \pi\left((m+1) y+(l+1) y^{-1}\right)\right) y^{\beta^{\prime}} d y \\
= & 2\left(\frac{m+1}{l+1}\right)^{-\frac{1}{2}\left(\beta^{\prime}+1\right)} K_{\beta^{\prime}+1}\left(4 \pi(l+1)^{\frac{1}{2}}(m+1)^{\frac{1}{2}}\right)
\end{aligned}
$$

and, putting $\beta^{\prime}=\beta, A_{1}^{\prime}$ is expressed as

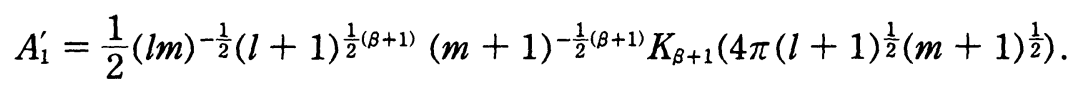

As for $A_{\tilde{Q}}^{\prime}, A_{Q}^{\prime}$ and $A_{\tilde{Q} Q}^{\prime},(16)$ yields

$$
\begin{aligned}
& \left|A_{\tilde{Q}}^{\prime}\right|<C_{4} l^{-1} A_{1}^{\prime}(\beta+1), \\
& \left|A_{Q}^{\prime}\right|<C_{4} m^{-1} A_{1}^{\prime}(\beta-1), \\
& \left|A_{\tilde{Q} Q}^{\prime}\right|<C_{4}^{2}(l m)^{-1} A_{1}^{\prime}(\beta),
\end{aligned}
$$

where $A^{\prime}(\boldsymbol{\beta})$ means $A_{1}^{\prime}$ as a function of $\beta$.

If (4) is applied to the above formula exressing $A_{1}^{\prime}$, there appears the formula in the proposition, and the order of $m$ in the formula is higher than the order of $m$ in the error term of (21) which expresses $A^{\prime}$. Furthermore, as (22) shows, the orders of $m$ in $A_{\tilde{Q}}^{\prime}, A_{Q}^{\prime}$, and $A_{\tilde{Q} Q}^{\prime}$ are lower than the order of $m$ in $A_{1}^{\prime}$ at least by $m^{\frac{1}{2}}$. This completes the proof.

Proposition 2.4. Let $s \in \mathbf{C}$, and let $h_{1}, h_{2}$ be as in (15). Then, provided that $s \neq \frac{1}{2}$, 


$$
\begin{gathered}
h_{1}(l, m) \sim 2^{-\frac{3}{2}}\left(\alpha\left(\bar{s}-\frac{1}{2}\right) l^{\bar{s}-\frac{1}{2}} m^{\frac{1}{2}\left(-\bar{s}+\frac{1}{2}\right)-1}\right. \\
\left.+\alpha\left(-\bar{s}+\frac{1}{2}\right) l^{-\bar{s}+\frac{1}{2}} m^{\frac{1}{2}\left(\bar{s}-\frac{1}{2}\right)-1}\right) \exp \left(-4 \pi m^{\frac{1}{2}}\right)
\end{gathered}
$$

as $m \rightarrow \infty$, and, for an arbitrary $s$,

$$
h_{2}(l, m) \sim 2^{-\frac{5}{2}} l^{-\frac{1}{2}}(l+1)^{-\frac{1}{4}} m^{-\frac{3}{4}} \exp \left(-4 \pi(l+1)^{\frac{1}{2}} m^{\frac{1}{2}}\right)
$$

as $m \rightarrow \infty$.

Proof. The result concerning $h_{1}$ follows from (13) and Proposition 2.1 with $\beta=0$. The result concerning $h_{2}$ follows from (14) and Proposition 2.3 with $\beta=-1$.

THEOREM 2.1. If $\sigma=\operatorname{Re} s>\frac{1}{2}$, then the asymptotic formula

$$
h(l, m) \sim 2^{-\frac{1}{2}} \alpha\left(-\bar{s}+\frac{1}{2}\right) l^{-\bar{s}+\frac{1}{2}} m^{\frac{1}{2}\left(\bar{s}-\frac{1}{2}\right)-1} \exp \left(-4 \pi m^{\frac{1}{2}}\right)
$$

holds for $h(l, m)$ in $(10)$ as $m \rightarrow \infty$. If $\sigma<\frac{1}{2}$, then the corresponding asymptotic formula is given by replacing $s$ with $1-s$ in the above formula. Moreover, if $s=\frac{1}{2}+i t,(t \in \mathbf{R}, t \neq 0)$, then we have

$$
\begin{aligned}
& h(l, m) \sim 2^{-\frac{1}{2}}\left(\alpha(i t) l^{i t} m^{-\frac{1}{2} i t-1}\right. \\
& \left.+\alpha(-i t) l^{-i t} m^{\frac{1}{2} i t-1}\right) \exp \left(-4 \pi m^{\frac{1}{2}}\right)
\end{aligned}
$$

as $m \rightarrow \infty$.

Proof. The order of $m$ is higher in $h_{1}$ than in $h_{2}$. Therefore, it is enough to prove the theorem for $h_{1}$ instead of $h$. If $\sigma>\frac{1}{2}$, then the order of $m^{\frac{1}{2}\left(\bar{s}-\frac{1}{2}\right)}$ is higher than the order of $m^{\frac{1}{2}\left(-\bar{s}+\frac{1}{2}\right)}$ so that the first formula in the theorem follows from (15) and Proposition 2.4. If $\sigma=\frac{1}{2}$, then the two orders are equal so that the second formula holds.

THEOREM 2.2. Let $h(l, m)$ be as in $(10)$, and let $s=\frac{1}{2}$. Then,

$$
h(l, m)>2 C_{0} m^{-1} \exp \left(-4 \pi m^{\frac{1}{2}}\right)
$$

holds for almost all $m$, where $C_{0}$ is the constant in Proposition 2.2. 
Proof. Proposition 2.2 with $\beta=0$ implies in this case

$$
h_{1}(l, m)>C_{0} m^{-1} \exp \left(-4 \pi m^{\frac{1}{2}}\right),
$$

while Proposition 2.4 is valid for $h_{2}$. Since $h_{1}$ contains $m$ in its highest order, the theorem is proved.

\section{§ 3. Main theorem}

Before presenting the main theorem, we state two propositions. The first one is fairly deep in which all results in Section 2 are concentrated, while the second one is an elementary and basic property of eigenfunctions of the Laplacian $D$.

PROPOSITION 3.1. Let $h(l, m)$ be as in (10) and (15), and let $\left\{c_{m}\right\}_{m=1}^{\infty}$ be a series of complex numbers such that the series $\sum_{m=1}^{\infty} h(l, m) c_{m}$ converges for all $l$. Then,

$$
c_{m}=o\left(m \exp \left(4 \pi m^{\frac{1}{2}}\right)\right) \text {. }
$$

Proof. Since $K_{\nu}=K_{-\nu}$, we may assume $\sigma \geqq \frac{1}{2}$. It follows from the convergence of the series that $c_{m}=o\left(h(l, m)^{-1}\right),(m \rightarrow \infty)$. Therefore, the proposition is an immediate consequence of the first asymptotic formula of Theorem 2.1 if $\sigma>$ $\frac{1}{2}$. If $\sigma>\frac{1}{2}$, then the proposition follows immediately from Theorem 2.2 .

Thus, in the rest of the proof, we may restrict ourselves in the case of $s=\frac{1}{2}+i t,(t \in \mathbf{R}, t \neq 0)$. Theorem 2.1 implies

$$
c_{m} \cdot\left(\alpha(i t) l^{i t} m^{-\frac{1}{2} i t}+\alpha(-i t) l^{-i t} m^{\frac{1}{2} i t}\right) m^{-1} \exp \left(-4 \pi m^{\frac{1}{2}}\right)=o(1) .
$$

So, putting $\alpha(i t)=\rho \exp (i t \theta),(\theta \in \mathbf{R})$, we have

$$
\alpha(i t) l^{i t} m^{-\frac{1}{2} i t}+\alpha(-i t) l^{-i t} m^{\frac{1}{2} i t}=2 \rho \cos t\left(\log \frac{m}{2}+\theta-\log l\right) .
$$

Now we choose $\varepsilon_{0}>0$ and a natural number $l_{0}$ such that $|\cos t x| \leqq \varepsilon_{0}$ implies $\left|\cos t\left(x-\log l_{0}\right)\right|>\varepsilon_{0},(x \in \mathbf{R})$, and look at $\cos t\left(\log \frac{m}{2}+\theta\right)$ which is a part of the right hand side of the above equality. If $\cos t\left(\log \frac{m}{2}+\theta\right)>\varepsilon_{0}$, then, with $l=1$, the absolute value of the left hand side of the above equality is bigger than $2 \rho \varepsilon_{0}$. If $\cos t\left(\log \frac{m}{2}+\theta\right) \leqq \varepsilon_{0}$, then, with $l=l_{0}$, the absolute value of the 
left hand side of the above equality is bigger than $2 \rho \varepsilon_{0}$. In either case, the theorem follows from (23).

Remark 3.1. If either $\sigma \neq \frac{1}{2}$ or $s=\frac{1}{2}$, then only one and arbitrary $l$ is enough in order to prove Proposition 3.1. If $\sigma=\frac{1}{2}$ and $s \neq \frac{1}{2}$, then any one $l$ and one more suitable $l_{0}$ are enough.

The next proposition is one of elementary properties of eigenfunctions of the Laplacian.

PROPOSITION 3.2. Let $\phi$ be a function on the upper half plane which is an eigenfunction of the Laplacian $D=y^{2}\left(\frac{\partial^{2}}{\partial x^{2}}+\frac{\partial^{2}}{\partial y^{2}}\right)$. Assume that $\phi(z)=\phi(-\bar{z})$ and $\phi(i y)=0$ for all $y>0$. Then, $\phi=0$ on $S$.

Proof. Put $D \phi=\lambda \phi$. Then, since

$$
\frac{\partial^{2 k} \phi}{\partial x^{2 k}}=\left(\lambda y^{-2}-\frac{\partial^{2}}{\partial y^{2}}\right)^{k},(k \geqq 1),
$$

$\left.\frac{\partial^{2 k} \psi}{\partial x^{2 k}}\right|_{x=0}$ is a linear combination of derivatives of various orders, and is 0 by the assumption. On the other hand, $\phi(z)=\phi(-\bar{z})$, i.e., $\phi(x+i y)=\phi(-x+i y)$ implies $\left.\frac{\partial^{2 k-1} \psi}{\partial x^{2 k-1}}\right|_{x=0}=0$. Hence, $\phi=0$.

Now we can prove the following main theorem:

THEOREM 3.1. Let $H$ be the infinite matrix given by (12), and $\left\{c_{m}\right\}_{m=1}^{\infty}$ be a series of complex numbers. Denote by $c_{*}$ the infinite column vector whose $m$-th component is $c_{m}$, and assume

$$
H c_{*}=0
$$

in the sense that $\sum_{m=1}^{\infty} h(l, m) c_{m}$ converges to 0 for every $l$. Then, $c_{m}$ are coefficients of an even cusp form as in (3).

Proof. Since Proposition 3.1 says $c_{m}=o\left(m \exp \left(4 \pi m^{\frac{1}{2}}\right)\right)$, (4) implies that

$$
f(z)=\sum_{m=1}^{\infty} c_{m} y^{\frac{1}{2}} K_{s-\frac{1}{2}}(2 \pi m y) \cos 2 \pi m x, \quad(z=x+i y),
$$


converges uniformly and absolutely in the subdomain of the upper half plane $S$ determined by $\operatorname{Im} s=y>y_{0}$, where $y_{0}>0$ is arbitrary. On the other hand, the formula

$$
-2 K_{\nu}^{\prime}(y)=K_{\nu-1}(y)+K_{\nu+1}(y)
$$

concerning the derivative of Bessel functions shows that the above series is still uniformly and absolutely convergent in the same domain even if each term is replaced by its derivatives of arbitrary orders with respect to $x$ as well as $y$. Therefore, the Laplacian $D$ can be applied termwise on the series so that

$$
D f=s(s-1) f .
$$

Next we consider the restriction of the function $f$ to the imaginary axis. Let $g_{m}(y)$ be as in (9). Then,

$$
f(i y)-f\left(i y^{-1}\right)=\sum_{m=1}^{\infty} c_{m} g_{m}(y)
$$

holds pointwise. At the same time, the assumption of the theorem implies that the series $\sum_{m=1}^{\infty} c_{m} g_{m}(y)$ converges weakly to 0 in the Hilbert space $\Omega$ introduced immediately prior to Proposition 2.1. Hence, it converges pointwise to 0 . This means

$$
f(i y)-f\left(i y^{-1}\right)=0 \text {. }
$$

Apply here Proposition 3.2 to $\phi(z)=f(z)-f\left(-z^{-1}\right)$. Then, we have $f(z)=f\left(-z^{-1}\right)$, from which follows that $f(z)$ is invariant under the operation of $\mathrm{SL}(2, \mathbf{Z})$.

This theorem shows that the Fourier coefficients of cusp forms are completely controlled by the infinite matrix $H$ which is constructed in a simple way by special functions.

\section{§ 4. Extended investigations including zeros of the Riemann zeta function}

By our investigations up to the preceding section it was shown that the matrix $H$ in (12) determines the Fourier coefficients of an even cusp form satisfying (1) as components of an infinite column vector $c_{*}$ annihilated by it. Namely, $c_{*}$ is an eigenvector of $H$ belonging to the eigenvalue 0 . Since $s$ is arbitrary, the existence of a cusp form belonging to $s$, or in other words, the fact that $s(s-1)$ is an eigenvalue of the Laplacian in the space of even automorphic functions with respect to $\operatorname{SL}(2 \mathbf{Z})$ which are square integrable on the fundamental domain, is equivalent to the fact that 0 is an eigenvalue of $H=H(s)$. 
Such a characterization of the eigenvalues of the Laplacian has a generalization in which the eigenvalues of the Laplacian and zeros of the Riemann zeta function are controlled simultaneously by an infinite matrix which is larger than the above $H$ by one row and by one column, while a crucial point is again whether or not the matrix has 0 as an eigenvalue. The aim of the present section is to explain this situation.

First we recall the notion of residual forms which are intimately connected with Eisenstein series. But, everything will be stated only under our restricted circumstances. For details, textbooks of Eisenstein series should be referred to.

A residual form means a function as in (2) with $c_{0}^{\prime}=0$ and $c_{0} \neq 0$, i.e., an automorphic form with a Fourier expansion of the form

$$
f(z)=c_{0} y^{1-s}+\sum_{m \neq 0} c_{m} y^{\frac{1}{2}} K_{s-\frac{1}{2}}(2 \pi|m| y) e(m x),
$$

$\left(c_{0} \neq 0\right)$. As mentioned at the beginning of Section 1 , the discontinuous group is always $\operatorname{SL}(2 \mathbf{Z})$.

For a point $z$ on the upper half plane $S$ and a complex number $s$ with Re $s>1$, the Eisenstein series $E(z, s)$ is defined by

$$
E(z, s)=\sum_{\substack{(c, d)=1 \\ c>0}} \frac{y^{s}}{|c z+d|^{2 s}}, \quad(c, d \in \mathbf{Z}) .
$$

This is an automorphic form, and its Fourier expansion is

$$
\begin{gathered}
E(z, s)=y^{s}+\phi(s) y^{1-s} \\
+\sum_{m \neq 0} 2 \pi^{s} \Gamma(s)^{-1} \delta(s, m) \zeta(2 s)^{-1} y^{\frac{1}{2}} K_{s-\frac{1}{2}}(2 \pi|m| y) e(m x),
\end{gathered}
$$

where $\zeta(s)$ is the Riemann zeta function,

$$
\phi(s)=\pi^{\frac{1}{2}} \frac{\Gamma\left(s-\frac{1}{2}\right)}{\Gamma(s)} \frac{\zeta(2 s-1)}{\zeta(2 s)},
$$

and

$$
\delta(s, m)=\sum_{d d^{\prime}=|m|}\left(\frac{d^{\prime}}{d}\right)^{s-\frac{1}{2}}=\delta(1-s, m), \quad(m \neq 0) .
$$

The function $E(z, s)$ has, as a function of $s$, a meromorphic continuation on the whole $s$-plane, and its poles coincide with those of $\phi(\mathrm{s})$ together with the orders. Furthermore, $E(z, s)$ satisfies (1), and is an even automorphic form because of $\delta(s, m)=\delta(s,-m)$.

Proposition 4.1. A pole $s_{0}$ of $\phi(s)$ is either $s_{0}=1$ or $s_{0}$ with $\zeta\left(2 s_{0}\right)=0$ and 0 $<\operatorname{Re} 2 s_{0}<1$. If $s_{0}$ is a pole of $\phi(s)$ of order $N$, then $\lim _{s \rightarrow s_{0}}\left(s-s_{0}\right)^{N} E(z, s)$ is a 
residual form.

Proof. The first assertion is a consequence of (26).

Let next $E(z, s)=\sum_{k=-N}^{\infty} f_{k}(z)\left(s-s_{0}\right)^{k}$ be the Laurent expansion of $E(z, s)$ at $s_{0}$. Then, since $E(z, s)$ satisfies (1), we have

$$
\begin{gathered}
\sum_{k=-N}^{\infty}\left(D f_{k}\right)(z)\left(s-s_{0}\right)^{k}=s(s-1) \sum_{k=-N}^{\infty} f_{k}(s)\left(s-s_{0}\right)^{k} \\
=\left(s_{0}\left(s_{0}-1\right)+\left(2 s_{0}-1\right)\left(s-s_{0}\right)+\left(s-s_{0}\right)^{2}\right) \sum_{k=-N}^{\infty} f_{k}(z)\left(s-s_{0}\right)^{k} .
\end{gathered}
$$

Hence, $D f_{-N}=s_{0}\left(s_{0}-1\right) f_{-N^{\prime}}$, and the constant term of the Fourier expansion of $f_{-N}$ is

$$
\lim _{s \rightarrow s_{0}}\left(s-s_{0}\right)^{N}\left(y^{s}+\phi(s) y^{1-s}\right)=c_{0} y^{1-s 0}
$$

with

$$
c_{0}=\lim _{s \rightarrow s_{0}}\left(s-s_{0}\right)^{N} \phi(s) .
$$

Therefore, $f_{-N}$ is a residual form, and certainly

$$
\lim _{s \rightarrow s_{0}}\left(s-s_{0}\right)^{N} E(z, s)=f_{-N}(z) .
$$

Here we recall so-called Maass-Selberg's inner product formula. Suppose that $f(z)=\sum a_{m}(y) e(m x)$ is an automorphic form satisfying $D f=\lambda_{a} f$, and define $f^{Y}(z)$ by

$$
f^{Y}(z)= \begin{cases}f(z)-a_{0}(y), & y>Y, \\ f(z), & y \leqq Y .\end{cases}
$$

On the other hand, put $\mathscr{D}=\left\{z \varepsilon S ;|z|>1,|\operatorname{Re} z|<\frac{1}{2}\right.$ ), which is a common fundamental domain of $\operatorname{SL}(2, \mathbf{Z})$. Then, provided that $g(z)=\sum b_{m}(y) e(m x)$ is an automorphic form satisfying $D g=\lambda_{b} g,\left(\lambda_{a} \neq \lambda_{b}\right)$,

$$
\int_{\mathscr{D}} f^{Y}(z) g^{Y}(z) \frac{d x d y}{y^{2}}=\frac{a_{0}{ }^{\prime}(Y) b_{0}(Y)-a_{0}(Y) b_{0}{ }^{\prime}(Y)}{\lambda_{a}-\lambda_{b}}
$$

holds, where $a_{0}^{\prime}$ and $b_{0}^{\prime}$ are derivatives of $a_{0}$ and $b_{0}$, respectively. This is Maass-Selberg's inner product formula which is fundamental in the theory of Eisenstein series. Its proof, being based upon Green's formula concerning the operator $\frac{\partial^{2}}{\partial x^{2}}+\frac{\partial^{2}}{\partial y^{2}}$, is not very difficult, but will not be given here. 
PROPOSITION 4.2. Every residual form belongs to a pole $s_{0}$ of the function $\phi(s)$ as considered in Proposition 4.1, and is given by the limit in Proposition 4.1 up to a constant factor.

Proof. First we show that $s_{0}$ must be a pole of $\Phi(s)$ whenever a residual form of the form $c_{0} y^{1-s_{0}}+\cdots$ exists. If we put $a_{0}(y)=c^{\prime} y^{s}+c y^{1-s}$ and $b_{0}(y)$ $=d^{\prime} y^{s^{\prime}}+d y^{1-s^{\prime}}$ in (27) with constants $c, c^{\prime}, d, d^{\prime}$, then the right hand side of (27) becomes

$$
\frac{c^{\prime} d^{\prime} Y^{s+s^{\prime}-1}-c d Y^{-s-s^{\prime}+1}}{s+s^{\prime}-1}+\frac{c^{\prime} d Y^{s-s^{\prime}}-c d^{\prime} Y^{-s+s^{\prime}}}{s-s^{\prime}}
$$

Assume now $f$ to be the Eisenstein series $E(z, s)=y^{s}+\phi(s) y^{1-s}+\cdots$ in (25) and $g$ to be a residual form of the form $y^{1-s 0}+\cdots$. Then, since $c^{\prime}=1, c=\phi(s)$, $d^{\prime}=0$, and $d=1,(27)$ implies

$$
\int_{\mathscr{D}} f^{Y}(z) g^{Y}(z) \frac{d x d y}{y^{2}}=\frac{-\phi(s) Y^{-s-s_{0}+1}}{s+s_{0}-1}+\frac{Y^{s-s_{0}}}{s-s_{0}}
$$

provided that $s \neq s^{\prime}=s_{0}$. Suppose here that $s_{0}$ is not a pole of $\phi(s)$. Then, unless $s_{0}=\frac{1}{2}$, the left hand side of the above formula is finite but the right hand side is infinite as $s \rightarrow s_{0}$, which is a contradiction. If $s_{0}=\frac{1}{2}$, then a direct computation shows $\phi\left(\frac{1}{2}\right)=-1$. Therefore, a similar contradiction takes place, too.

To prove next the last half of the proposition, it is enough to show that there exists at most one residual form for an $s_{0}$ up to a constant factor. Assume that there are two residual forms of the form $y^{1-s_{0}}+\cdots$. Then, their difference is a cusp form. But, as stated in Proposition 4.1, a pole of $\phi(s)$ is either $s_{0}=1$ or $s_{0}$ with $\zeta\left(2 s_{0}\right)=0$ and $0<\operatorname{Re} 2 s_{0}<1$. To such an $s_{0}$, no cusp forms can belong. In fact, the former case is known in the investigations on the least absolute value of the eigenvalues of the Laplacian. In the latter case, $\operatorname{Re} s_{0} \neq \frac{1}{2}$ and $s_{0}$ is not real, and so $s_{0}\left(s_{0}-1\right)$ cannot be an eigenvalue of an elliptic operator.

Since the Eisenstein series $E(z, s)$ is an even automorphic form, Proposition 4.2 immediately implies the following:

Proposition 4.3. Every residual form is an even automorphic form. 
After these preparations, we construct a matrix $H_{0}$ which is larger than $H$ in (12) by one row and by one column. To do this, we put

$$
g_{0}(y)=y^{1-s}-y^{-1+s}
$$

and

$$
H_{0}=H_{0}(s)=(h(l, m))_{l, m=0}^{\infty}
$$

using the definition (10) of $h(l, m)$ unchanged for $l, m=0$, too. The matrix $H_{0}$ is hermitian as well as $H$.

Let $m>0$ and $s \in \mathbf{C}$, and put, analogously to (13) and (14),

$$
\begin{aligned}
h_{1}(0, m) & =\int_{0}^{\infty} y^{1-\bar{s}} K_{s-\frac{1}{2}}(2 \pi m y) \omega(y) y^{\frac{1}{2}} \frac{d y}{y} \\
& =\int_{0}^{\infty} K_{s-\frac{1}{2}}(2 \pi m y) \omega(y) y^{\frac{1}{2}-\bar{s}} d y \\
h_{2}(0, m) & =\int_{0}^{\infty} y^{-1+\bar{s}} K_{s-\frac{1}{2}}(2 \pi m y) \omega(y) y^{\frac{1}{2}} \frac{d y}{y} \\
& =\int_{0}^{\infty} K_{s-\frac{1}{2}}(2 \pi m y) \omega(y) y^{-\frac{3}{2}+\tilde{s}} d y .
\end{aligned}
$$

Then, analogously to (15), we have

$$
h(0, m)=2 h_{1}(0, m)-2 h_{2}(0, m) .
$$

The following proposition gives an asymptotic formula of $h(0, m)$ as $m \rightarrow \infty$ in a corresponding form to Propositions 2.1 to 2.3:

Proposition 4.4. Let $s^{\prime} \in \mathbf{C}$ and $\beta \in \mathbf{C}$. Then,

$$
\int_{0}^{\infty} K_{s^{\prime}-\frac{1}{2}}(2 \pi m y) \omega(y) y^{\beta} d y=2^{-\frac{3}{2}} m^{-\frac{\beta+2}{2}} \exp \left(-4 \pi m^{\frac{1}{2}}\right)\left(1+O\left(m^{-\frac{1}{2}}\right)\right)
$$

holds as $m \rightarrow \infty$.

Proof. Denote by $B$ the integral in the proposition, i.e.,

$$
B=\int_{0}^{\infty} K_{s^{\prime}-\frac{1}{2}}(2 \pi m y) \omega(y) y^{\beta} d y .
$$

Divide this integral as $\int_{0}^{\frac{1}{m}}+\int_{\frac{1}{m}}^{\infty}$. Then, $\omega(y)<\exp \left(-2 \pi y^{-1}\right)$ yields $\int_{0}^{\frac{1}{m}}$ $=O(\exp (-2 \pi \gamma m))$ for an arbitrary $\gamma$ with $0<\gamma<1$. Therefore, applying (4) to $\int_{\frac{1}{m}}^{\infty}$, we see 


$$
\begin{gathered}
B=\frac{1}{2} m^{-\frac{1}{2}} \int_{0}^{\infty} \exp \left(-2 \pi\left((m+1) y+y^{-1}\right)\right) y^{\beta-\frac{1}{2}}\left(1+Q\left((m y)^{-1}\right)\right) d y \\
+O(\exp (-2 \pi r m),
\end{gathered}
$$

where $Q$ is as in (16). Denote by $B_{1}$ and $B_{Q}$ the contributions to the integral of terms in the sum $1+Q$, respectively. Then,

(18) with $\beta^{\prime}=\beta-\frac{1}{2}$ furnishes

$$
B_{1}=2\left(\frac{1}{m+1}\right)^{\frac{1}{2}\left(\beta+\frac{1}{2}\right)} K_{\beta+\frac{1}{2}}\left(4 \pi(m+1)^{\frac{1}{2}}\right)
$$

On the other hand, (18) with $\beta^{\prime}=\beta-\frac{3}{2}$ similarly shows that the order of $m$ in $B_{Q}$ is lower than the order of $m$ in $B_{1}$ by $m^{\frac{1}{2}}$. Thus, the proposition follows from (4).

Proposition 4.5. The following two asymptotic formulas hold as $m \rightarrow \infty$ :

$$
\begin{aligned}
& h_{1}(0, m) \sim 2^{-\frac{3}{2}} m^{\frac{\bar{s}}{2}-\frac{5}{4}} \exp \left(-4 \pi m^{\frac{1}{2}}\right), \\
& h_{2}(0, m) \sim 2^{-\frac{3}{2}} m^{-\frac{\bar{s}}{2}-\frac{1}{4}} \exp \left(-4 \pi m^{\frac{1}{2}}\right) .
\end{aligned}
$$

Proof. The first formula follows from (29) and from Proposition 4.4 with $\beta=\frac{1}{2}-\bar{s}$. The second formula follows from (30) and from Proposition 4.4 with $\beta=-\frac{3}{2}+\bar{s}$

THEOREM 4.1. Let $s \in \mathbf{C}$ and $\sigma=\operatorname{Re} s$. Then, as $m \rightarrow \infty$,

$$
h(0, m) \sim 2^{-\frac{1}{2}} m^{\frac{s}{2}-\frac{5}{4}} \exp \left(-4 \pi m^{\frac{1}{2}}\right)
$$

holds if $\sigma>1$, and

$$
h(0, m) \sim-2^{-\frac{1}{2}} m^{-\frac{s}{2}-\frac{1}{4}} \exp \left(-4 \pi m^{\frac{1}{2}}\right),
$$

holds if $\sigma>1$. If $\sigma=1$, then $h(0, m)$ has the asymptotic value which is the sum of the right hand sides of the two formulas.

Proof. The theorem follows from Proposition 4.5, whenever $\frac{\sigma}{2}-\frac{5}{4}$ and $-\frac{\sigma}{2}-\frac{1}{4}$ are compared. 
Preceding investigations assure us that asymptotic estimations of $h(l, m)$ from below and above corresponding to Proposition 1.2, Theorem 2.1, and in Theorem 2.2 exist for $H_{0}$, too. Consequently, the proofs of Theorem 1.1 and Theorem 3.1 are applicable to $H_{0}$ and to residual forms, too. We are, however, exclusively dealing with even automorphic forms so that every automorphic form in our consideration is of the form

$$
f(z)=c_{0} y^{1-s}+\sum_{m=1}^{\infty} c_{m} y^{\frac{1}{2}} K_{s-\frac{1}{2}}(2 \pi m y) \cos 2 \pi m x
$$

instead of (24). The results are summed up in the following:

THEOREM 4.2. Let $H_{0}$ be as in (28), let $\left\{c_{m}\right\}_{m=0}^{\infty}$ be a series of complex numbers, and let $c_{*}$ be the infinite column vector whose $m$-th component is $c_{m}$. Then, $H_{0} c_{*}=0$ in the sense that $\sum_{m=0}^{\infty} h(l, m) c_{m}$ converges to 0 for all $l$ is equivalent to the fact that $c_{m}$ are Fourier coefficients of an even automorphic form as in (31). The automorphic form is a cusp form if $c_{0}=0$, and is a residual form if $c_{0} \neq 0$.

This theorem, together with Propositions 4.1 to 4.3 , implies

THEOREM 4.3. Let $H_{0}=H_{0}(s)$ be as in (28). Then, there exists an infinite vector such that $H_{0} c_{*}=0$ if and only if one of the following three conditions is satisfied: a) $s=1, \mathrm{~b})$ there exists a cusp form with respect to $\mathrm{SL}(2, \mathbf{Z})$ belonging to the eigenvalue $s(s-1)$, c) $2 s$ is a zero of the Riemann zeta function in the critical strip.

Remark 4.1. The last half of Proposition 4.2 is a different expression of the fact that a residual form and a cusp form cannot belong to one and the same eigenvalue. Therefore, no two of the three cases a), b), and c) in Theorem 4.3 can occur at the same time.

\section{BIBLIOGRAPHY}

[1] D. A. Hejhal, The Selberg trace formula and the Riemann zeta function, Duke Math. J., 43-3 (1976), 441-482.

[2] T. Kubota, Elementary of Eisenstein series, Kodansha Scientific, 1972.

[3] J.-M. Deshouillers and H. Iwaniec, Kloosterman sums and Fourier coefficients of cusp forms, Invent. Math., 70 (1982), 219-288.

Department of Mathematics

Nagoya University

Chikusaku Nagoya, 464-01

Japan 